\title{
Grundsatz des gleichen Zuganges zu den Leistungen der Gesundheitsfürsorge
}

\author{
Daniel Eryk Lach
}

\section{Der verfassungsrechtliche Grundsatz als Programmnorm}

Der Grundsatz des gleichen Zuganges zu den Leistungen der Gesundheitsfürsorge ist das Fundament (die Leitidee) des polnischen Systems der Gesundheitsfürsorge. Er ist in Art. 68 Abs. 2 der polnischen Verfassung (pV) verankert, demzufolge die öffentliche Gewalt den Staatsangehörigen, unabhängig von deren materiellen Lage, gleichen Zugang zur Gesundheitsfürsorge, die aus öffentlichen Mitteln finanziert wird, zusichert. Bedingungen und Umfang der Leistungen regelt das Gesetz.

Bei der Auslegung der Vorschrift des Art. 68 Abs. $2 \mathrm{pV}$ ist vor allem die Tatsache zu berücksichtigen, dass die sozialen Rechte und Freiheiten, welche mit den Richtungen und Zielen der von der öffentlichen Gewalt geführten Sozialpolitik verbunden sind, besonderen Rechtscharakter haben. Wie Garlicki anmerkte, muss die Verfassung in Bezug auf die Verhältnisse zwischen Staat und Bürger die Normen nicht so präzise bestimmen, dass diese ohne Hilfe von weiteren Normen oder Auslegungsinstrumenten niedrigerer Ordnung angewendet werden könnten. Dies folgt aus der Tatsache, dass das Grundgesetz in der Sprache von Werten, Grundsätzen und Ideen geschrieben ist und keine konkret formulierten Rechtsnormen enthält. ${ }^{1}$

Im Lichte der Aussagen der Verfassungsrechtliteratur scheint es richtig, anzunehmen, dass Art. 68 Abs. 2 pV Programmcharakter hat, d.h. dass die Vorschrift ihre Adressaten durch die Vorgabe der gewünschten Ziele bindet, aber keine konkreten und notwendigen Ausführungsschritte bestimmt. Sie bezieht sich auf ein allgemeines gesellschaftliches Wissen ihrer Adressaten, das seinerseits eine empirische Grundlage besitzt. Die in solchen

1 Vgl. Garlicki, Normatywna wartość ustawy zasadniczej (Normative Wert des Grundgesetzes), in: Wyrzykowski (Hrsg.), Konstytucyjne podstawy systemu prawa (Verfassungsrechtliche Grundsätze des Rechtssystems), Warschau 2001, S. 19. 
Normen vorgegebenen Ziele haben einen verbindlichen Charakter in dem Sinne, dass sie so verwirklicht werden müssen, dass die verpflichteten Subjekte zumindest von der Sorge um Beibehaltung des erreichten Status quo befreit würden. ${ }^{2}$

Im Kontext des Programmcharakters des Art. 68 Abs. 2 pV lassen sich - bezugnehmend auf die Literatur - auch folgende Argumente hervorheben: 1. Die Vorschrift enthält eindeutige Vorgaben für das Gesetz zur Bestimmung des Inhalts der Berechtigung (Art und Umfang von Leistungen $)^{3}$. 2. Die Vorschrift bezieht sich auf die Ziele des Normgebers und nicht des Normadressaten, sie ge- oder verbietet dem Adressaten, d.h. der

2 Vgl. Sarnecki, Normy programowe w Konstytucji i odpowiadające im wolności obywatelskie (Programnormen in der Verfassung und die den entsprechenden Bürgerfreiheiten), in: Garlicki/Szmyt (Hrsg.), Sześć lat Konstytucji Rzeczypospolitej Polskiej. Doświadczenia i inspiracje (Sechs Jahre der Verfassung von Republik Polen. Erfahrungen und Inspirationen), Warschau 2003, S. 252 ff. Auch in der deutschen Literatur wird angeführt, dass bei Programmnormen „dem Gesetzgeber eine bestimmte Richtung für seine Aktivität zwecks Annäherung an bestimmte Sachziele vorgeschrieben wird”. „Bei den Programmen geht es [...] um eine in die Zeit hinein wirkende, sich kontinuierlich erprobende Linie". Vgl. Lerche, Das Bundesverfassungsgericht und die Verfassungsdirektiven, AöR 1965, S. 347, zu den ,nicht erfüllten Gesetzgebungsaufträgen“.

3 Vgl. Sarnecki (Fn. 2), S. 254; Garlicki merkte hierin an, dass - weil die Verfassung als anwendbares Recht gedacht wurde - bei der Formulierung von Sozialrechten mehrere Klauseln eingeführt worden sind, welche die ,Zulässigkeit der Bestimmung von Begrenzungen in Bezug auf einzelne Rechte und Freiheiten“ vorsehen. In diesem Lichte ist die Vorschrift des Art. 68 Abs. 2 Satz 2 der Verfassung zu verstehen, wonach Bedingungen und Umfang der Leistungen das Gesetz regelt. Garlicki deutete darauf hin, dass die sehr allgemeine Formulierung von Sozialrechten und die Übergabe der Kompetenz, deren Inhalt und Umfang zu bestimmen, an den Gesetzgeber, ein bewusster Akt der Verfassungsautoren war, da sie Bürgeransprüche befürchteten, welche das Gleichgewicht der öffentlichen Finanzen stören könnten (vgl. Garlicki, Wolności i prawa jednostki w Konstytucji Rzeczypospolitej Polskiej z 1997 roku. Bilans pięciu lat (Freiheiten und Rechte der Einheit in der Verfassung der Republik Polens vom 1997. Bilanz von 5 Jahren), in: Jerzmański (Hrsg.), Pięć lat Konstytucji Rzeczypospolitej Polskiej. Materiały z konferencji na Zamku Królewskim w Warszawie, 17 października 2002 (Fünf Jahre der Verfassung der Republik Polens. Materialien von der Konferenz am Königsschloss in Warschau, 17. Oktober 2002), Warschau 2002, S. 61 und 68 f. In diesem Lichte ist auch auf die Aussage von Jellinek hinzuweisen, wonach „Die Begründung eines subjektiven Rechts [...] in der Regel nicht [...] durch Rechtsätze, welche Prinzipien für [die] Erlassung künftiger oder Auslegung bestehender Gesetze enthalten" stattfindet. Vgl. Jellinek, System der subjektiven öffentlichen Rechte, Tübingen 1905, S. $71 \mathrm{f}$. 
öffentlichen Gewalt, also unmittelbar kein Handeln zur Erreichung des Ziels. ${ }^{4}$ 3. Das Ziel ist nicht als empirisch identifizierbarer Stand der Dinge bestimmt - zum ersten, weil in Art. 68 Abs. 2 pV Wertaussagen verwendet werden (der „gleiche“ Zugang) ${ }^{5}$, und zum zweiten, weil die Pflicht der Sicherung des gleichen Zuganges als ,regulative Idee“ zu verstehen ist, die nie vollkommen verwirklicht werden kann. ${ }^{6}$ Wie Zacher bereits bemerkte: „Mehr Gleichheit in der Sicherheit bedeutet zugleich neue Ungleichheit.“7

Bei der Bestimmung des Rechtscharakters des Art. 68 Abs. 2 pV als Programmnorm ist auch die Aussage von Banaszak hervorzuheben, der feststellte, dass in dieser Vorschrift das Ziel des Handelns der öffentlichen Gewalt deutlich bestimmt wurde, wobei die Norm nicht Programm-, sondern Gewährleistungscharakter hat. Die öffentliche Gewalt ist verpflichtet, die entsprechenden Institutionen zu schaffen, welche der Vorbeugung von Krankheiten, und im Falle des Auftretens von Krankheiten deren Behandlung dienen werden. ${ }^{8}$ Den normativen Charakter dieser Pflicht bestätigend konstruierte Banaszak anstelle einer Programmnorm die Gewährleistungsnorm.

4 Vgl. Gizbert-Studnicki/Grabowski, Normy programowe w Konstytucji Programmnormen in der Verfassung in: Trzciński (Hrsg.), Charakter i struktura norm Konstytucji (Charakter und Struktur der Verfassungsnormen), Warschau 1997, S. 98. Die Feststellung, welches Handeln von bestimmten Subjekten (un-)zulässig ist, damit das in der Programmnorm definierte Ziel erreicht werden kann, ist möglich. Es geschieht allerdings nur im Wege von juristischen Schlussfolgerungen (effet utile, effet nécessaire), welche sich auf das empirische Wissen der Kausalzusammenhänge beziehen, was über den Gesetzestext hinausgeht.

5 Wobei bei der Bewertung der Gleichheit des Zugangs die Grundsätze der (sozialen) Gerechtigkeit und Solidarität berücksichtigt werden müssen.

6 Vgl. Gizbert-Studnicki/Grabowski (Fn. 4), S. 99 f.

7 Vgl. Zacher, Abhandlungen zum Sozialrecht, Heidelberg 1993, S. 34.

8 Vgl. Banaszak, Konstytucja Rzeczypospolitej Polskiej. Komentarz, (Verfassung der Republik Polens. Kommentar) Warschau 2009, Kommentar zu Art. 68 Rn. 4. 
Es scheint die These berechtigt zu sein, dass dieses Konzept sich auf die Idee des Gewährleistungsstaats ${ }^{9}$ (the enabling state ${ }^{10}$ ) bezieht. Auf deren Grundlage wird auch die Technik ${ }^{11}$ der treuhänderischen Verwaltung als eine Art der Verwirklichung der Idee der sozialen Sicherung in Bezug auf die Gesundheitsfürsorge vorgesehen. Der Nationale Gesundheitsfonds als Organisator des polnischen Systems der Gesundheitsfürsorge ist im Lichte seiner Aufgaben und seines Rechtscharakters weder ein Sozialversicherungsträger noch ein Organ der Leistungsverwaltung. Im Grunde genommen handelt er wie ein treuhänderischer Verwalter ${ }^{12}$, der in eigenem Namen, aber für die Leistungsberechtigten, die Verträge für die Erbringung der Leistungen der Gesundheitsfürsorge aushandelt, abschließt und abrechnet. Damit erfüllt der Fonds die öffentlichen Aufgaben aus dem Bereich der sozialen Sicherheit, die ihm durch den Gesetzgeber auferlegt worden sind. Bezugnehmend auf die Aussagen der polnischen Literatur ${ }^{13}$

9 Mehr dazu: Franzius, Der „Gewährleistungsstaat“ - ein neues Leitbild für den sich wandelnden Staat?, Der Staat 42 (2003), S. 493-517; ders., Gewährleistung im Recht. Grundlagen eines europäischen Regelungsmodells öffentlicher Dienstleistungen, Tübingen 2009, S. 24 ff., 39; Luthe, Der aktivierende Sozialstaat im Recht, Nachrichtendienst Deutscher Verein 2003, Nr 5, S. 167; Kurzke-Maasmeier, Aktivierende Soziale Arbeit im reformierten Sozialstaat, in: Kurzke-Maasmeier/ Mandry/Oberer (Hrsg.), Baustelle Sozialstaat! Sozialethische Sondierungen in unübersichtlichem Gelände, Münster 2006, S. 111-128.

10 Vgl. Jończyk, Trudna modernizacja opieki zdrowotnej w Niemczech i w Zjednoczonym Królestwie (UK) (Schwierige Modernisierung der Gesundheitsfürsorge in Deutschland und in der Vereinigten Königreich), PiZS 2006, Nr 6, S. 6.

11 In der polnischen Literatur gilt als herrschende Meinung (die an die deutsche Rechtstheorie von vor 30. Jahren anknüpft), dass die Idee der sozialen Sicherheit mit diversen „Methoden“ oder „Techniken“ verwirklicht werden kann: Fürsorge (z.B. Sozialhilfe), Versicherung (z.B. Gesetzliche Krankenversicherung) und Versorgung (z.B. staatlicher Gesundheitsdienst). Vgl. z.B. Schulin, Techniken und Instrumente der sozialen Sicherung, in: v. Maydell/Hohnerlein (Hrsg.), Die Umgestaltung der Systeme sozialer Sicherheit in den Staaten Mittel- und Osteuropas, Fragen und Lösungsansätze, Berlin 1993, S. 174; ebenso Kaczyński, Pojęcie zabezpieczenia społecznego i kryterium rozróżniania jego technik (Der Begriff der sozialen Sicherheit und Kriterium für die Unterscheidung ihre Techniken), PiZS 1986, Heft 5-6, S. 20-31.

12 Dazu eingehender: Lach: Powiernicza technika realizacji zabezpieczenia społecznego (na przykładzie ubezpieczenia zdrowotnego) (Treuhänderische Technik der Verwirklichung der sozialen Sicherheit (am Beispiel der Krankenversicherung), „Państwo i Prawo” 2009, Heft 3, S. 34-47.

13 Vgl. Biernat, Prywatyzacja zadań publicznych. Problematyka prawna (Privatisierung von öffentlichen Aufgaben. Rechtsproblematik), Warschau - Krakau 1994. 
ist darin die Privatisierung öffentlicher Aufgaben als Instrument des Gewährleistungsstaates zu sehen. ${ }^{14}$ Der Staat leistet nicht alleine (mittels der Leistungsverwaltung), sondern organisiert den rechtlichen Rahmen des Systems und bestimmt die Aufgaben nichtstaatlicher Rechtssubjekte, welche dann die öffentlichen Aufgaben für die öffentliche Gewalt vornehmen und ausführen. ${ }^{15}$

Des Weiteren stellte Banaszak fest, dass aus der in Art. 68 Abs. 2 der Verfassung verankerten Pflicht der öffentlichen Gewalt keine subjektiven Rechte ableitbar sind. ${ }^{16}$ Auch nach Jończyk begründet Art. 68 Abs. 2 pV keine Ansprüche von Versicherten, sondern enthält im Grunde lediglich ein Verbot der Differenzierung von Bürgern nach dem Kriterium der materiellen Lage. ${ }^{17}$

Diese Ansicht vertrat auch (unmittelbar auf Art. 68 Abs. 3 pV bezogen $^{18}$ ) der polnische Verfassungsgerichtshof (Trybunał Konstytucyjny, TK). Im Urteil vom 22. Juli $2008^{19}$ hielt er fest, dass aufgrund dieser Vorschrift die Programmnorm konstruiert werden kann, sie aber keine Rechte bestimmt, welche dem Bürger unmittelbar zustehen und - als „Vorschrift in der Gestalt eines Staatspolitikgrundsatzes und nicht eines Bürgerrechts“" - weder individuelle Anspruchsgrundlage, noch Quelle von verfassungsrechtlichen subjektiven Rechten sein kann. ${ }^{20}$ Der Gerichtshof deutete da-

14 Vgl. Lach, Prawny paradygmat opieki zdrowotnej a stosunek „ubezpieczenia zdrowotnego" i jego charakter prawny (Das Rechtsparadigma der Gesundheitfürsorge und das Verhältnis der „Krankenversicherung” und seinen Rechtscharakter), in: $Z$. Kubot, T. Kuczyński (Hrsg.), Współczesne zagadnienia prawa pracy i prawa socjalnego. Księga Jubileuszowa z okazji 70-tych urodzin Profesora Herberta Szurgacza (Gegenwärtige Fragen des Arbeits- und Sozialrechts. Jubiläumsbuch zum 70. Geburtstag von Professor Herbert Szurgacz), Breslau 2011, S. 451-463.

15 Vgl. Lach, Powierzanie realizacji zadań publicznych z zakresu zabezpieczenia społecznego podmiotom niepublicznym, (Anvertrauen der Verwirklichung von öffentlichen Aufgaben aus dem Bereich der sozialen Sicherung den nichtöffentlichen Subjekten), „Państwo i Prawo” 2015, Heft 2, S. 35-57.

16 Vgl. Banaszak (Fn. 8), Kommentar zu Art. 68, Rn. 10.

17 Vgl. Jończyk, Glosse zum Urteil des Obersten Gerichts (Sąd Najwyższy - SN) v. 12.02.2004 r., II UK 243/03, OSP 2004, Heft 10, S. 130.

18 Wonach die öffentliche Gewalt verpflichtet ist, den besonderen Schutz der Kinder, Schwangeren, Behinderten und Älteren zu sichern.

19 VerfGH v. 22.07.2008, K 24/07.

20 Vgl. auch TK v. 12.11.2003 r., Ts 11/03, OTK-B 2003, Nr 4, Pos. 226. Es ist aber zu beachten, dass der Gerichtshof in früheren Judikaten eine andere Meinung vertreten hat. In Oberstes Gericht v. 23.03.1999, K 2/98 stellte der Gerichtshof fest, dass von Art. 68 Abs. 1 der Verfassung (Jeder hat das Recht auf Gesundheits- 
bei darauf hin, dass dieser Gedankengang an die Erwägungen von Verfassungsautoren anknüpft. ${ }^{21}$

Zusammenfassend kann gesagt werden, dass aus der Vorschrift des Art. 68 Abs. 2 (in Verbindung mit Abs. 3) pV die Pflicht der öffentlichen Gewalt folgt, ein System der Gesundheitsfürsorge aufzubauen, welches einerseits das Gleichheitsprinzip in Bezug auf den Zugang zu den Leistungen der Gesundheitsfürsorge verwirklicht, andererseits ausgewählten Subjekten besondere Vorsorge sichert. Die Konstruktion des Art. 68 Abs. 2 $\mathrm{pV}$ und dessen Trennung in zwei Sätze - wovon der erste die Pflicht der öffentlichen Gewalt statuiert, den gleichen Zugang zu den Leistungen der Gesundheitsfürsorge zu sichern, und der zweite es dem Gesetzgeber gebietet, die Bedingungen und den Umfang der Leistungen in dem entsprechenden Gesetz zu regeln - erlaubt den Schluss, dass man aufgrund des Art. 68 Abs. 2 pV kein Recht auf den gleichen Zugang zu den Leistungen der Gesundheitsfürsorge, die aus den öffentlichen Mitteln finanziert wird, als öffentliches subjektives Recht konstruieren darf. Die ausgeführte Rechtsnorm hat dazu auch die innere Struktur eines Prinzips ${ }^{22}$, d.h. sie hat den Charakter einer Optimierungsnorm, in welcher der ideale Zustand, der so weit wie möglicherreicht werden soll, beschrieben ist. Das rechtsanwendende Organ ist somit an ein bestimmtes normatives Ideal gebunden. Eine solche Norm gebietet die Verwirklichung des gleichen Zuganges zu den Leistungen im maximalen Grad, wobei die rechtlichen und tatsächlichen Möglichkeiten der Verwirklichung berücksichtigt werden müssen, also mit dem Vorbehalt der Zulassung einer bestimmten Differenzierung.

schutz) „das subjektive Recht des Einzelnen auf den Gesundheitsschutz und das objektive Gebot für die öffentliche Gewalt, die Handlungen vorzunehmen, welche für den entsprechenden Schutz und die Verwirklichung dieses Rechtes notwendig sind, abzuleiten ist.“ Diese Meinung wurde später in TS v. 10.01.2001, Ts 72/00, OTK 2001, Nr 1, Pos. 12 bestätigt. Sie folgt aber den anderen Inhalt und Charakter von Abs. 1 und Abs. 2 des Art 68 der Verfassung.

21 Vgl. Eckhardt, Prawo do ochrony zdrowia. Zagadnienia wybrane (Recht auf Gesundheitsschutz. Ausgewählte Fragen), Prawa Człowieka 2006, Nr. 10, S. 67 f.

22 Vgl. Alexy, Rechtsregeln und Rechtsprinzipien, Archiv für Rechts- und Sozialphilosophie 25 (1985), S. 13-29; ders.: Theorie der Grundrechte, Baden-Baden 1985; ders.: Zum Begriff des Rechtsprinzips, Rechtstheorie 1 (1979), S. 63 ff.; GizbertStudnicki, Zasady i reguły prawne (Rechtsregeln und Rechtsprinzipien), „Państwo i Prawo" 1988, Heft 3, S. 17; Gizbert-Studnicki/Grabowski (Fn. 4), S. 101 ff.; Lerche: Das Bundesverfassungsgericht und die Verfassungsdirektiven. $\mathrm{Zu}$ den ,nicht erfüllten Gesetzgebungsaufträgen”, AöR 90 (1965) S. 347; Sarnecki (Fn. 2), S. 252-254. 
Aus der so verstandenen Pflicht der öffentlichen Gewalt kann man dann als Reflex ein subjektives Rechts ableiten. Dieser Reflex verbietet es, die Rechte so auszugestalten, dass daraus eine ungerechtfertigte Differenzierung des Zuganges zu den Leistungen der Gesundheitsfürsorge folgt, was vor allem die Einführung von ungerechtfertigten Finanzsperren beim $\mathrm{Zu}$ gang zu den Leistungen betrifft. Im Übrigen schützt Art. 68 Abs. 2 pV einen inhaltlichen Kern (core). Als Kern (Minimum) kann dann die Pflicht der öffentlichen Gewalt, das Gesundheitsfürsorgesystem zu schaffen, angesehen werden. Dabei gilt: Das System muss aus öffentlichen Mitteln finanziert sein (d.h. es muss ein öffentlich-rechtlicher Mechanismus für die Ansammlung und die Ausgabe von Mitteln geschaffen werden). Zudem muss der gleiche Zugang zu den Leistungen der Gesundheitsfürsorge für alle Staatsangehörigen (tatsächlich für alle Berechtigten) gesichert sein, d.h. Quantität und Qualität (Standard) der Leistungen und die Grundsätze der Erbringung können nicht von der materiellen Lage oder dem Grad der finanziellen Teilnahme (Beitragsleistungen) eines Berechtigten abhängig sein, wenn auch die Kostenteilnahme typisiert und unterschiedlich sein darf - Ausschluss der Risikoselektion. Es ist damit auch eine solche Ausgestaltung verboten, welche den Zugang zu den Leistungen der Gesundheitsfürsorge, in Bezug auf die materielle Lage der berechtigten Bürger unbegründet differenzieren würde, was vor allem die Einführung von ungerechtfertigten Finanzschwellen betrifft.

\section{Die Umsetzung der Programmnorm}

1. Sicherung des gleichen Zugangs zu den Leistungen der Gesundheitsfürsorge als verfassungsrechtliche Pflicht der öffentlichen Gewalt

In Art. 68 Abs. 2 pV wurde „die Sicherung des gleichen Zugangs zu den Leistungen der Gesundheitsfürsorge" als Aufgabe der öffentlichen Gewalt formuliert. Der Begriff „Sicherung“ ist hier anders als „,soziale Sicherung“ zu verstehen. Hier geht es eher um „das Sichern“ oder - bezugnehmend auf die schon erwähnte Idee vom Gewährleistungsstaat - ,die Gewährleistung". Die Vorschrift knüpft nämlich an den seit einiger Zeit zu beobachtenden Prozess der Übergabe (oder des Anvertrauens) von öffentlichen Aufgaben (oder deren Verwirklichung) aus dem Bereich der sozialen Sicherung (oder sogar der Sozialpolitik) an nichtöffentliche Subjekte an. Zur 
Durchführung wird dabei kein öffentlich-rechtliches, sondern das privatrechtliche Instrumentarium verwendet.

Diese Form der Privatisierung wurde bereits in der Literatur diskutiert. Szurgacz gab zu bedenken, dass sich ein Rückzug des Staates in Bezug auf die Erbringung von Sozialleistungen und den Einsatz von nichtöffentlichen Subjekten in diesem Gebiet beobachten lässt. ${ }^{23}$ Dieses Phänomen wurde vom zitierten Autor gerade mit dem Konzept des Gewährleistungsstaates, welcher als ,dritter Weg zwischen dem klassischem Interventionsund dem Wohlstandstaat" bezeichnet worden ist, erklärt. ${ }^{24}$ Die in der deutschen Literatur ${ }^{25}$ ausgearbeitete Definition des Gewährleistungsstaates präzisiert, dass „der Gewährleistungsstaat der Staat ist, der an seiner konkreten Gemeinwohlverantwortung festhält, aber die Instrumente zur eigenhändigen, also unmittelbaren Aufgabenerfüllung aufgegeben hat. Er führt den prinzipiellen sozialgestalterischen Anspruch des wohlfahrtsorientierten Erfüllungsstaates in dem Sinne fort, dass er auf bestimmte positive gesellschaftliche Verhältnisse und Zustände zielt und sich weiterhin in der Letztverantwortung hierfür sieht.“

In diesem Zusammenhang ist auch die Privatisierung von öffentlichen Aufgaben aufzuführen, durch die ein Staat (die öffentlichen Gewalt) von einem selbstständigen Leistungserbringer zu einem Garanten für die Aus-

23 Jończyk wies dabei auf die Unterscheidung zwischen dem klassischen privatrechtlichen „Hartvertrag“ (hard contracting) und dem neuen „Weichvertrag“ (soft contracting) hin, wobei es bei dem letzten Konstrukt vor allem um die Anknüpfung der festen Kooperation zwischen Parteien geht, um dem gemeinsamen Ziel der Parteien zu folgen. Soft contracting bezieht sich dann vor allem auf die Situationen, in denen das öffentliche Subjekt als Disponent von öffentlichen Mitteln dominiert. Vgl. Jończyk, Glosse zum Urteil des Obersten Gerichts (Sąd Najwyższy SN) v. 10.12.2004 r., III CK 134/04, OSP Nr 6/2005, S. 333; Treutner, Kooperativer Rechtsstaat. Das Beispiel Sozialverwaltung, Baden-Baden 1998.

24 Szurgacz, Zagadnienie przekazywania przez państwo zadań w zakresie zabezpieczenia społecznego podmiotom niepublicznym (Die Frage der Übergabe vom Staat den Aufgaben aus dem Bereich der sozialen Sicherung durch den Staat an den nichtöffentlichen Subjekten) in: Szurgacz (Hrsg.), Konstytucyjne problemy prawa pracy i zabezpieczenia społecznego. Referaty na XV Zjazd Katedr i Zakładów Prawa Pracy i Ubezpieczeń Społecznych, Wrocław 1-2 czerwca 2005 r.(Verfassungsprobleme des Arbeits- und Sozialsicherungsrechtes. Referaten für die 15. Tagung von Lehrstühlen und Anstalten des Arbeits- und Sozialversicherungsrechtes, Wrocław 1-2.06.2005), Breslau 2005, S. 125-142.

25 Vgl. Franzius (Fn. 9), S. 494; ders., Die Europäische Dimension des Gewährleistungsstaates, Der Staat 2006 (45), S. 547. 
führung der öffentlichen Aufgaben wird ${ }^{26}$, wobei diese „Garantie“ (richtig: Gewährleistung) vor allem durch den Aufbau der Rechtsregulation geleistet ist. ${ }^{27}$ Von großer Bedeutung sind auch die pragmatischen Fragen, da die nichtöffentlichen Subjekte oft effektiver arbeiten als die öffentlichen Einrichtungen. ${ }^{28}$

Wenn es um den Sinn des Gewährleistungsstaates und die daraus folgenden Aufgaben/ Pflichten der öffentlichen Gewalt geht, ist festzustellen, dass diese Gewährleistung nicht nur die Haftung für nichtöffentliche Subjekte wegen der fehlerhaften Ausübung der ihnen übertragenen Aufgaben nach sich zieht, sondern auch die entsprechenden Präventionsmaßnahmen umfasst. $^{29}$ Der aktivierende Staat ${ }^{30}$ darf selbst nicht passiv bleiben. Dies ist insbesondere darauf zurückzuführen, dass es hier um öffentliche Aufgaben geht, die für die gesamte Gesellschaft von großer Bedeutung sind.

In diesem Kontext sollte auch die der öffentlichen Gewalt auferlegte Pflicht der „Sicherung von gleichem Zugang zu den Leistungen der Gesundheitsfürsorge" verstanden werden. Es geht um mehr als nur die Regulierung von Rechtsverhältnissen zwischen Akteuren des Systems der Gesundheitsfürsorge, was die in Art 68 Abs. 2 Satz 2 pV bestimmte Aufgabe des Gesetzgebers ist. Die „Sicherung“ als Gewährleistung umfasst vielmehr auch die Pflicht der Exekutive, die Kontroll- und Aufsichtstätigkeit gegenüber dem Nationalen Gesundheitsfonds (NFZ) und den Leistungserbringern (sowohl individuellen Vertretern von medizinischen Berufen, welche die wirtschaftliche Tätigkeit selbständig als Freiberufler oder in der Gruppe als Partnergesellschaft ausüben, als auch juristischen Personen

26 Vgl. Biernat (Fn. 13), S. 7 ff. In der deutschen Literatur wurde dazu, ausgehend von der Idee geteilter Verantwortung, die Gewährleistung als Gegenbegriff zur Erfüllung bestimmt. Wo dem Staat eine Gewährleistungsverantwortung zugewiesen sei, schließe dies die Übernahme von Erfüllungsverantwortung aus, vgl. Ruge, Die Gewährleistungsverantwortung des Staates und der Regulatory State, Berlin 2004, S. 180 .

27 Vgl. Franzius (Fn. 9), S. 499, 510-515.

28 Szurgacz (Fn. 24), S. 132 f.

29 Vgl. Franzius (Fn. 9), S. 496 ff., 504 ff.; ders., Gewährleistungsrecht als Erscheinungsform des Verwaltungsrechts, Zeitschrift für öffentliche und gemeinwirtschaftliche Unternehmen, ZögU 2008, S. 372 ff.

30 Luthe, Der aktivierende Sozialstaat im Recht, Nachrichtendienst Deutscher Verein 2003, Nr. 5, S. 167-177; Kurzke-Maasmeier, Aktivierende Soziale Arbeit im reformierten Sozialstaat, in: Kurzke-Maasmeier/Mandry/Oberer (Hrsg.), Baustelle Sozialstaat! Sozialethische Sondierungen in unübersichtlichem Gelände, Münster 2006, S. 111-128. 
jeder Art die gesetzesgemäß die medizinische Tätigkeit ausüben) auszuüben. Die Aufsicht über das Funktionieren des Systems ist dabei dynamisch zu verstehen, d.h. nicht nur mit Blick auf die Erfüllung der gesetzlichen Anforderungen, sondern im Kontext der Umsetzung des Grundsatzes des gleichen Zugangs. Diese Dynamik begründet u.a. die Notwendigkeit einer regelmäßigen Evaluierung der geltenden Rechtsregelungen und der Übereinstimmung der Wirkungen ihrer Anwendung, mit dem Ziel, kontinuierlich die erforderlichen Anpassungen vornehmen zu können, um das System zu optimieren. ${ }^{31}$ Es ist zu bedenken, dass die in Art. 68 Abs. 2 pV verankerte Pflicht der öffentlichen Gewalt nicht nur als gesetzgeberische Kompetenz zu verstehen ist. Hierin ist das Ziel des Gesetzgebers definiert, welches als Optimierungsgebot die Richtung der gesetzgeberischen Aktivität bestimmt. Da als Ziel die Sicherung des gleichen Zugangs festgelegt wurde, ist bei der Bewertung der Verwirklichung dieser Vorgabe die Problematik des Gleichheitsgrundsatzes im Sozialrecht zu betrachten. Zum einen geht es hier um die Frage der gerechtfertigten Differenzierung zwischen der Rechtslage von bestimmten Subjekten bzw. Personengruppen in Bezug auf den Zugang zu den Leistungen der Gesundheitsfürsorge (hiermit sind sowohl der Zugang zum System, die Eintrittsbedingungen, z.B. Beitragssatz, als auch der Zugang innerhalb des Systems, d.h. die Inanspruchnahmebedingungen, z.B. Eigenanteil an den Kosten der Leistungen der Gesundheitsfürsorge, gemeint). Zum anderen folgt daraus die Pflicht der ständigen und kontinuierlichen Weiterentwicklung der praktizierten Lösungen, um die derzeitigen und künftigen Ungleichheiten zu überwinden, denn noch immer gilt: „Mehr Gleichheit in der Sicherheit bedeutet zugleich neue Ungleichheit". 32

Die postulierte Gleichheit des Zuganges bezieht sich laut Art. 68 Abs. 2 $\mathrm{pV}$ auf die materielle Lage von Bürgern. In diesem Sinne verdient das Urteil des polnischen Verfassungsgerichthofes vom 7. Januar $2004^{33}$ Beachtung, in dem festgestellt wurde, dass es in Art. 68 Abs. 2 pV ,um den gleichen Zugang für alle Bürger unabhängig von deren materieller Lage geht", da „die Grundsätze der Inanspruchnahme von Leistungen der Gesundheitsfürsorge vom Grad der Teilnahme der einzelnen Mitglieder der Gemeinschaft an den öffentlichen Mitteln, die die Finanzierungsquelle dieser

31 Vgl. Lach, Organisation, Akteure und Rechtsverhältnisse im polnischen System der Gesundheitsfürsorge, ZIAS 2016, Heft 2, S. 289-301.

32 Vgl. Zacher (Fn. 7), S. 34.

33 VerfGH v. 07.01.2004, K 14/03. 
Leistungen darstellen, unabhängig sind." Der Gesetzgeber muss das System so organisieren, dass weder der Zugang zum System (Systemeinbeziehung und Beginn der Garantiephase) noch der Zugang zu den Leistungen der Gesundheitsfürsorge (bei Beginn der Realisationsphase beim Eintritt des Sozialrisikos) von der materiellen Lage, insbesondere von dem Finanzierungsgrad, abhängig ist. Wie Jończyk anführte, liegt der Sinn der Pflicht des Gesetzgebers aus Art. 68 Abs. 2 pV darin, angesichts ,der momentan bestehenden beträchtlichen sozialen Unterschiede in Polen die Gefahr der Risikoselektion in der Gesundheitsfürsorge zu minimieren." ${ }^{34}$ Es ist dabei zu betonen, dass die Gleichheit des Zuganges nicht unmittelbar, sondern durch das Versicherungsverhältnis (Verhältnis zwischen dem Versicherten und dem Nationalen Gesundheitsfonds) und das Organisationsverhältnis (Verhältnis zwischen den Leistungserbringern und dem Nationalen Gesundheitsfonds) realisiert werden soll. ${ }^{35}$

Des Weiteren lässt sich festhalten, dass aufgrund des Inhalts und des Rechtscharakters von Art. 68 Abs. 2 pV (Programmnorm mit der Struktur des Optimierungsgebotes) das in dieser Vorschrift definierte Ziel Gleichheit des Zuganges - optimal verwirklicht werden soll. Die Maximierung von Leistungen darf hingegen nicht als von der Verfassung angestrebtes Ziel verstanden werden. Priorität hat die Unabhängigkeit des $\mathrm{Zu}$ ganges $\mathrm{zu}$ den Leistungen der Gesundheitsfürsorge von der materiellen Lage der Bürger. Daraus folgt, dass sowohl die Konstruktion des Systems (Systemeinbeziehung- und Finanzierungsbedingungen) als auch der Garantieumfang (Quantität und Qualität von Leistungen) Instrumente des sozialen Ausgleiches sein sollen. ${ }^{36}$

34 Jończyk, Ochrona zdrowia (Gesundheitschutz), PiP 2007, Heft. 2, S. 6.

35 Vgl. Jończyk, Stosunek opieki zdrowotnej (Gesundheitsfürsorgeverhältnis), PiZS, Nr. 3/2007, S. 5.

36 Vgl. Lach, Komercjalizacja SPZOZ-ów a obowiązki władzy publicznej w zakresie zapewnienia równego dostępu do świadczeń opieki zdrowotnej, część 1 (Kommerzialisierung von „selbständigen öffentlichen Gesundheitsfürsorgeanstalten“ und die Pflichten der öffentlichen Gewalt im Bereich der Sicherung des gleichen Zugangs zu den Leistungen der Gesundheitsfürsorge, Teil 1), PiZS 2012, Heft 9, S. 2-8. 


\section{Sicherung des gleichen Zugangs und Rechtfertigung einer Differenzierung}

Wie schon in der Literatur ${ }^{37}$ dargestellt, muss man bei der Analyse des Grundsatzes des gleichen Zugangs zu den Leistungen der Gesundheitsfürsorge berücksichtigen, dass dieser Grundsatz die Sozialrechtsregulierung betrifft, welche die Verwirklichung von sozialpolitischen Zielen bezweckt und sich deswegen auf eine eigene Axiologie stützt. Da das Sozialrecht Chancengleichheit und die Nivellierung übermäßiger Ungleichheiten in der Lebenssituation verschiedener Personengruppen anstrebt, müssen seine Normen von Anfang an die Ungleichheit in Bezug auf die anerkannten Rechte oder auferlegten Lasten, die Differenzierungen also, berücksichtigen. Dabei muss im Einklang mit der polnischen Lehre und Rechtsprechung des polnischen Verfassungsgerichtes die Bewertung, inwieweit die Vorschriften, die das System der Gesundheitsfürsorge konstituieren und regulieren, die Grundsätze des gleichen Zugangs und der gleichen Betrachtung verwirklichen, zweistufig vorgenommen werden. Zunächst sind die Verteilungskriterien zu beurteilen, die zur Differenzierung hinsichtlich der Rechtsstellung bestimmter Subjektgruppen herangezogen wurden. Diese sollten objektiv, relevant, verhältnismäßig und ausreichend begründet sein. Anschließend wird die in der Norm angewandte Verteilung von Rechten und Pflichten auf die zuvor bestimmten Personengruppen dahingehend untersucht, inwieweit sie bestehende Unterschiede berücksichtigt und damit gerecht ist.

Für die Feststellung, ob die Typisierung ausgewählter Subjektgruppen und die Differenzierung hinsichtlich ihrer Rechtsstellung ,gerecht“ ist, ist die Begründetheit des angenommenen Differenzierungskriteriums zu überprüfen. Diese soll aus dem angenommenen Wertesystem sowie den Verfassungsgrundsätzen oder -normen folgen. Als erstes muss in diesem Kontext der (soziale) Solidaritätsgrundsatz herangezogen werden, der die Basis und das Leitprinzip des Versicherungssystems als eine von mehreren Methoden der Verwirklichung der Idee der sozialen Sicherung dar-

37 Vgl. Lach, Zasada równego dostępu do świadczeń opieki zdrowotnej (Grundsatz des gleichen Zugangs zu den Leistungen der Gesundheitsfürsorge), Warschau 2011, S. 81 ff.;ders. Die Verteilungsgerechtigkeit in der Gesundheitsfürsorge, in: Classen/Lukańko/Richter (Hrsg.), Diskriminierung aufgrund der Gesundheit in alternden Gesellschaften. Deutschland und Polen vor neuen Herausforderungen, Berlin 2015, S. 281-292. 
stellt. Der Solidaritätsgrundsatz betrifft vor allem die Pflichten der Systemteilnehmer (sowie Benefiziare) und nicht deren Rechte. ${ }^{38}$ Daraus folgt, dass man die Teilnahme an der Risikogemeinschaft und die Höhe des Beitrags zu ihrer Finanzierung im Lichte der Solidarität bewerten kann. Das Recht auf Leistungen folgt bereits aus der Systemteilnahme selbst. Sein Umfang richtet sich nach dem individuellen Gesundheitszustand (Bedarf). Der Solidaritätsgrundsatz braucht hier nicht angewendet zu werden. Der Inhalt des Grundsatzes der sozialen Solidarität (Solidarprinzip) ist der, dass die aus den bestehenden Interdependenzen folgende Übernahme von bestimmten Lasten und Pflichten durch die Gemeinschaft einen sozialen Ausgleich innerhalb der Gemeinschaft herbeiführt. Die Idee der sozialen Solidarität ist also die Vergemeinschaftung („Entindividualisierung“) des Risikos. ${ }^{39}$ Dies wird vor allem in der Konstruktion des Beitrages ersichtlich ist, der ausschließlich von der Einkommenshöhe und nicht von dem Risiko oder Bedarf des Einzelnen abhängt (interpersonaler Ausgleich). ${ }^{40}$

An anderer Stelle thematisiere ich das Verhältnis zwischen Gleichheitsund Gerechtigkeitsgrundsatz. ${ }^{41}$ Ich komme zu dem Schluss, dass die Gleichheitsregel in der Tat nur eine der Gerechtigkeitsregeln ist, da sowohl die gleiche (identische - jedem das Gleiche oder gleich viel) als auch ungleiche (differenzierte) Verteilung gerecht sein kann. ${ }^{42}$ Es ist jedoch zu bedenken, dass im Falle der legislativen Aktivitäten der öffentlichen Ge-

38 Vgl. Lach, O solidarności społecznej w ,ubezpieczeniu zdrowotnym” (Von der sozialen Solidarität in der „Krankenversicherung), in: Skapski/Ślebzak (Hrsg.), Aksjologiczne podstawy prawa pracy i ubezpieczeń społecznych (Axiologische Grundsätze des Arbeits- und Sozialversicherungsrechtes), Posen 2014, S. 271-285.

39 Die Vergemeinschaftung (,Entindividualisierung“) des Risikos unterscheidet die soziale Versicherung von den privatrechtlichen Versicherungen, bei welchen die Höhe der Beiträge grundsätzlich von dem individuellen Risiko des Versicherten abhängt.

40 Vgl. Kirchhof, Das Solidarprinzip im Sozialversicherungsbeitrag, in: Schulin (Hrsg.), Sozialfinanzverfassung: 5. Sozialrechtslehrertagung 6. bis 8. März 1991 in Göttingen, Wiesbaden 1992, S. 65 ff.

41 Vgl. Lach, Die Verteilungsgerechtigkeit in der Gesundheitsfürsorge, in: Classen/ Łukańko/Richter (Hrsg.), Diskriminierung aufgrund der Gesundheit in alternden Gesellschaften. Deutschland und Polen vor neuen Herausforderungen, Berlin 2015, S. 281-292.

42 Vgl. Nowacki, Sprawiedliwość a równość w orzecznictwie Trybunału Konstytucyjnego (Die Gerechtigkeit und die Gleichheit in der Rechtsprechung des Verfassungsgerichtes), in: Kudej (Hrsg.), W kręgu zagadnień konstytucyjnych. Profesorowi Eugeniuszowi Zwierzchowskiemu w darze (Im Kreis der Verfassungsfragen. Festgabe für Professor Eugene Zwierzchowski), Kattowitz 1999, S. 83-103. 
walt, vor allem im Sozialrecht, kaum eine Situation vorstellbar ist, in der eine einheitliche Entscheidung über die Verteilung von Gütern, Rechten oder Pflichten möglich wäre. Schließlich folgt jede Gesellschaftspolitik der unbestrittenen These von der bestehenden Diversität der Lebens- und Daseinskonditionen der verschiedenen sozialen Gruppen. Wenn eines der Grundziele der Gesellschaftspolitik die Nivellierung von übermäßigen Disproportionen in diesem Bereich sein soll, dann muss eine diesem Ziel dienende Regelung von einer Ungleichheit in Bezug auf die zuerkannten Rechte oder auferlegten Lasten ausgehen. Erst im Lichte dieser Annahme kann man die bestehende Regelung im Hinblick auf die angewandten Verteilungskriterien, anhand derer (zwischen den Subjekten) differenziert wurde, bewerten. Dabei ist zu prüfen, ob sie objektiv, relevant, verhältnismäßig und gerecht, d. h. geeignet, ist, um feststellen zu können, ob die Verteilung von Rechten und Pflichten zwischen den so bestimmten Personengruppen gleich, also in dem Sinne gerecht ist, dass die bestehenden Unterschiede berücksichtigt wurden.

Aus der Anerkennung der Verteilungsgerechtigkeit als begründetem und relevantem Ziel des Rechts folgt die Frage, ob es einen Konflikt zwischen dem vom Rechtssystem verwirklichten Gerechtigkeitsgrundsatz und dem Grundsatz der Gleichheit vor dem Gesetz geben kann. Jede Forderung nach einer differenzierten Behandlung, die aus der materiellen Gerechtigkeit abzuleiten sein kann (z.B. Sonderpflege für gesellschaftlich benachteiligte Gruppen oder Bevorzugung von ausgewählten Subjektgruppen, ausgedrückt in Sonderleistungsberechtigungen [Leistungen außerhalb des Katalogs, außerhalb der Reihe, ohne Zuzahlung u.a.]), scheint der Gleichheit vor dem Gesetz zu widersprechen. In diesem Kontext ist jenen Literaturaussagen beizupflichten, denen zur Folge der Begriff der Gleichheit vor dem Gesetz keinen autonomen, von anderen Werturteilen unabhängigen, Inhalt hat und der Grundsatz der Gleichheit vor dem Gesetz inhaltlich sekundär gegenüber den Grundsätzen der materiellen Gerechtigkeit ist und daher nicht deren Zulässigkeitskriterium sein kann. ${ }^{43}$

Dies heißt also auch, dass wenn der Gleichheitsgrundsatz keinen absoluten Charakter hat, die Differenzierung nach der jeweiligen Rechtssituation zulässig ist, sofern sie nicht - mangels hinreichender Begründung -

43 Vgl. Sadurski, Teoria sprawiedliwości. Podstawowe zagadnienia, (Die Theorie der Gerechtigkeit, Grundfragen), Warschau 1988, S. 87 f.; ähnlich: Nowacki (Fn. 42), S. 95, 103. 
eine Diskriminierung oder unzulässige Bevorzugung darstellt. ${ }^{44}$ Mit anderen Worten hat die Gleichheit im Recht materiellen und nicht formellen Charakter, sie betrifft die Begründetheit der Wahl des Differenzierungskriteriums, welche aus dem angenommenen Wertsystem folgt. ${ }^{45}$ Auch das Verfassungsgericht war der Auffassung, dass die Differenzierung im Recht zulässig ist, soweit sie gerechtfertigt ist. ${ }^{46}$

\section{Verwirklichung des Grundsatzes des gleichen Zugangs zu den Leistungen der Gesundheitsfürsorge}

Die Analyse der Gleichheit des Zuganges zu den Leistungen der Gesundheitsfürsorge betrifft also die Frage, ob Differenzierungen aufgrund der unterschiedlichen Rechtssituationen bestimmter leistungsberechtigter Gruppen im Lichte des Gerechtigkeitsgrundsatzes (in verschiedenen Aspekten durch das Solidarprinzip korrigiert) begründet sind, nicht aber den tatsächlichen (räumlichen, technischen u.a.) Zugang. Dabei soll der Zugang zu den Leistungen der Gesundheitsfürsorge als die Möglichkeit der Nutzung der im System garantierten Gesundheitsfürsorge(-leistungen) verstanden werden, was sowohl den Zugang zum System, als auch den Zugang zu der Gesundheitsfürsorge (bzw. den -leistungen) für die Systemteilnehmer umfasst. Der Gegenstand des Zugangs (und der Finanzierung) ist die Gesundheitsfürsorge, deren Umfang in jedem Fall individuell angepasst wird, wobei die erforderlichen medizinischen Dienst- und Sachleistungen von der Fachbewertung des medizinischen Personals abhängig sind. Die Bewertung von bestehenden sozialrechtlichen Regelungen soll,

44 Vgl. Garlicki, Zasada równości i zakaz dyskryminacji w orzecznictwie Trybunału Konstytucyjnego (Gleichheitsgrundsatz und Diskriminierungsverbot in der Rechtsprechung des Verfassungsgerichthofes), in: Oliwa-Radzikowska (Hrsg.), Obywatel - jego wolności i prawa. Zbiór studiów przygotowanych z okazji 10-lecia urzędu Rzecznika Praw Obywatelskich (Bürger - seine Freiheiten und Rechte. Sammlung von Studien vorbereitet zum 10. Jubiläum des Ombudsman-Amtes), Lodz 1998, S. 66.

45 Vgl. Sadurski, Równość wobec prawa (Gleichheit im Recht), PiP 1978, Nr. 8-9, S. 58 f. und die dort zitierte Literatur; auch: Sadurski (Fn. 43), S. 98 f.

46 Vgl. VerfGH v. 26.09.1989, K 3/89, OTK 1989, Nr. 1, Pos. 5; v. 08.05.1990, K 1/90, OTK 1990, Nr. 1, Pos. 2; v. 22.08.1990, K 7/90, OTK 1990, Nr. 1, Pos. 5; v. 28.05.1991, K 1/91, OTK 1991, Nr. 1, Pos. 4; v.07.01.1992, K 8/91, OTK 1992, Nr. 1, Pos. 5 i v. 28.11.1995, K 17/95, OTK 1995, Nr. 3, Pos. 18. 
soweit es deren Vereinbarkeit mit dem Grundsatz des gleichen Zugangs betrifft, einen abstrakt-rechtlichen und keinen konkreten Charakter haben. Bewertungsgegenstand ist die den Zugang betreffende rechtliche Regelung und nicht der Zugang als solcher in seiner räumlichen und technischen Bedeutung, der sich auf die individuelle Situation eines konkreten Leistungsempfängers bezieht. Es ist selbstverständlich und kann nicht im Lichte des Gleichheitssatzes bewertet werden, dass die Bewohner größerer Städte besseren (leichteren) Zugang zu den, vor allem stationären oder fachärztlichen, Gesundheitsfürsorgeleistungen haben, als die Versicherten aus ländlichen Regionen.

Soweit es die subjektive Seite der Verwirklichung des Grundsatzes des gleichen Zugangs zu den Leistungen der Gesundheitsfürsorge betrifft, ist anzunehmen, dass - angesichts des breiten, aber doch begrenzten Katalogs von zum Systembeitritt verpflichteten Subjekten - deren Erfüllung drei Instrumente dienen. Der Zugang zum System ist damit auch für die nicht versicherungsverpflichteten Personen gesichert. Es geht hier um die freiwillige Versicherung, die aber wegen der Höhe der Beitragsgrundlage und des Zusatzbeitrags grundsätzlich auf die wohlhabenderen Personen (wenn auch ohne Einkommen, z.B. Rentner) begrenzt ist. In diesem Sinne findet die freiwillige Versicherung kaum praktische Anwendung. Zu dem Kreis der Leistungsberechtigten wurden auch Personen ohne Versicherungstitel, die die polnische Staatsangehörigkeit besitzen und ihren Wohnsitz im Gebiet von Polen haben, gezählt, deren Einkünfte die von Art. 8 des Gesetzes über die Sozialhilfe genannten Grenzen nicht überschreiten und gleichzeitig die in Art. 7 dieses Gesetzes genannten Voraussetzungen für die Erteilung von Sozialhilfe erfüllen. Die genannten Personen sind nicht in der Lage, ihre schwierige Lebenssituation mit Hilfe ihrer eigenen Ressourcen zu überwinden. Mit der Einbeziehung auch der ärmsten Bürger, für die keine Beitragspflicht besteht, folgt der Gesetzgeber dem Gebot, die Leistungen der Gesundheitsfürsorge für alle Bürger, unabhängig von deren materiellen Lage, gleich zugänglich zu machen. Im Unterschied zu den Versicherten werden die Leistungen für diese Berechtigten nicht aus Mitteln des Fonds, sondern aus der Staatskasse finanziert. Zum dritten sind auch Personen ohne Versicherungstitel, die ihren Wohnsitz im Gebiet von Polen haben und nicht älter als 18 Jahre oder schwanger sind, leistungsberechtigt., Dies gilt auch während der Geburt bzw. dem Wochenbett. Auch hier gilt natürlich keine Beitragspflicht. Zusammenfassend kann gesagt werden, dass Leistungsberechtigte in der Tat alle Bürger sind - um die Vorgabe des Art. 68 Abs. 2 der Verfassung zu erfüllen. 
Es ist auch zu erwähnen, dass das Gesetz Sonderberechtigungen für bestimmte Subjekte vorsieht. Diese Frage wurde bereits im Schrifttum diskutiert ${ }^{47}$, daher soll hier lediglich festgestellt werden, dass weder die Bestimmung dieser Subjekte noch deren Bevorzugung hinsichtlich der Rechte auf Einwände im Hinblick auf den Grundsatz des gleichen Zugangs zu den Leistungen der Gesundheitsfürsorge stößt und die Regelungen somit als gerecht(fertigt) bezeichnet werden können. Fraglich sind aber die Art, wie diese Leistungen finanziert werden, sowie die Belastung der Gemeinschaft der Leistungsberechtigten mit den daraus folgenden Kosten. Dies gilt umso mehr, als es bei den Bevorzugten meistens um ein Recht auf Leistungen geht, das an die Idee der sozialen Entschädigung anknüpft (Leistungen für die Kriegsversehrten u.a.).

Soweit es die objektive Seite der Verwirklichung des Grundsatzes des gleichen Zugangs $\mathrm{zu}$ den Leistungen der Gesundheitsfürsorge angeht, muss zuerst zwischen dem Recht des Leistungsberechtigten auf die weit verstandene Gesundheitsfürsorge, welche aus öffentlichen Mitteln finanziert sein soll, und dem Recht auf bestimmte Leistungen der Gesundheitsfürsorge differenziert werden. Die abstrakte Definition der Gesundheitsfürsorge ist, im Interesse des Nationalen Gesundheitsfonds (wegen seiner Organisationspflichten) und der Leistungserbringer (im Hinblick auf das Entgelt), in den von diesen Parteien abgeschlossenen „Verträgen über die Erbringung der Leistungen der Gesundheitsfürsorge" konkretisiert. Diese Verträge dürfen aber natürlich nicht den Umfang der Ansprüche der Leistungsberechtigten auf die Leistungen der Gesundheitsfürsorge bestimmen - dies folgt aus dem Gesetz. Der Katalog von garantierten Leistungen, in dem nicht nur die einzelnen Leistungen, sondern auch die Konditionen für deren Erfüllung und Finanzierung bestimmt sind, dient sowohl der eindeutigen Feststellung des Umfangs von Leistungen, welche im System finanziert und im Rahmen der gesetzlichen Garantie erfüllt werden, als auch der Vereinheitlichung der oben erwähnten Verträge. Damit sollen territoriale Unterschiede des Leistungsumfangs sowie der -konditionen ausgeschlossen werden. Bezugnehmend auf die finanziellen Begrenzungen und das Bedürfnis, das Verhalten der Leistungsberechtigten und -erbringer zu steuern, sehen die Verträge, für bestimmte Leistungen auch (Anzahl- oder Betrags-) Grenzen vor. Der Zugang zu diesen Leistungen muss reglementiert werden, dies erfolgt durch die Verwendung von Wartelisten. Dadurch

47 Vgl. Lach (Fn. 41), S. 281-292. 
werden allerdings die Rechte der Leistungsberechtigten (Umfang der gesetzlichen Garantie) begrenzt bzw. die Leistungsberechtigten werden unterschiedlich behandelt. Die Möglichkeit, bestimmte Leistungen in Anspruch zu nehmen, wird zeitlich verschoben, d.h. die Leistungsberechtigten müssen auf einen freien Termin warten. Begründet wird dies mit dem Gemeinwohlprinzip: Die Knappheit der Finanzmittel, die dem Nationalen Gesundheitsfonds zur Verfügung stehen, rechtfertigt die Limitierung der Möglichkeit der Leistungsinanspruchnahme, da das Interesse aller Leistungsberechtigten, eine bestimmte Leistung unter Garantie erhalten zu können, gegenüber dem Interesse eines einzelnen Patienten, diese Leistung unverzüglich (auf Anfrage) in Anspruch nehmen zu können, überwiegt. Damit wird auch an die Systemgrundlagen angeknüpft - denn dieses bezieht sich auf die Rechte der Leistungsberechtigten (im rechtlichem Sinne) und nicht der Patienten (im faktischem Sinne).

Hinsichtlich finanzieller Aspekte des Grundsatzes des gleichen $\mathrm{Zu}-$ gangs zu den Leistungen der Gesundheitsfürsorge ist zwischen der Frage der Beitragsfinanzierung der Systemgarantie (nebst Einschluss in der Versichertengruppe Subjekten, die keine Einkünfte erreichen und keine Beiträge bezahlen) und der Problematik des Selbstanteiles in der Verwirklichungsphase zu unterscheiden. Bei der Regelung der öffentlichen Abgabe sui generis, im Gesetz Beitrag genannt, wird zwischen verschiedenen Subjektgruppen erheblich und unverhältnismäßig differenziert. Das Fehlen einer Beitragsbemessungsgrenze führt zusätzlich zu der Differenzierung zwischen einzelnen Personen innerhalb der jeweiligen Gruppe. Eine solche Differenzierung lässt sich nicht mit dem Grundsatz der sozialen Solidarität rechtfertigen. Die Einbeziehung von Personen, die selbst kein Einkommen haben und deren Beitrag von der Staatskasse finanziert wird (wobei dies umstritten ist), in das Versicherungssystem könnte mit den Grundsätzen der sozialen Gerechtigkeit (Art. $2 \mathrm{pV}$ ) begründet werden. Problematisch ist die Belastung von Patienten mit den (Teil-)Kosten, die bei der Inanspruchnahme bestimmter Sach- und Begleitleistungen auferlegt werden, wenn es um individualisierte Leistungen geht. Die individuelle materielle Lage wird dabei nicht berücksichtigt, was zur Verfassungswidrigkeit dieser Regelung führen könnte. In diesem Zusammenhang ist auf das Gemeinwohlprinzip hinzuweisen, welches den Vorrang der Interessen der Versichertengemeinschaft gegenüber den Interessen des einzelnen Patienten zum Ausdruck bringt. Besondere Bedeutung kommt dabei den Vorschriften des neuen Arzneimittelkostenrückerstattungsgesetzes zu, in denen die materielle Lage der Versicherten in höherem Maße Beach- 
tung findet als in der früheren Regelung. Die Rückerstattungsstufen hängen nicht mehr von medizinischen Kriterien (Art der Arzneimittel Grund-, Ergänzungs- oder sonstige Mittel), sondern von finanziellen Kriterien (Kosten der Therapie etc.) ab. Damit wird das Gebot des Art. 68 Abs. $2 \mathrm{pV}$ beachtet und in einem volleren Maß zur Verwirklichung gebracht. ${ }^{48}$

\section{Fazit}

1. Art. 68 Abs. 2 der Verfassung hat den Charakter einer Optimierungsnorm im Sinne der möglichst umfassenden Verwirklichung eines idealen Zustands. Die das Recht anwendenden Organe sind daher an ein bestimmtes normatives Ziel gebunden und sollen eine umfassende Verwirklichung des gleichen Zugangs zu den Leistungen unter Beachtung der rechtlichen und tatsächlichen Möglichkeiten gewährleisten. In diesem Rahmen und unter bestimmten Voraussetzungen ist eine Differenzierung zulässig.

2. Daraus wiederum folgt die Pflicht der öffentlichen Gewalt, die bestehende Rechtsregelung zu überwachen und die bestehenden Lösungen kontinuierlich an die tatsächliche Situation anzupassen, um die derzeitigen und zukünftigen Ungleichheitszustände in Bezug auf den $\mathrm{Zu}-$ gang zu den Leistungen der Gesundheitsfürsorge zu beseitigen oder zumindest zu begrenzen.

3. Kern des Grundsatzes des gleichen Zugangs ist dann ein an die öffentliche Gewalt gerichtetes Gebot, ein von öffentlichen Mitteln finanziertes System der Gesundheitsfürsorge aufzubauen. Dieses System soll als wichtigste Vorgabe die Gleichheit des Zugangs ermöglichen. Gleichzeitig muss aber, wegen des Inhalts des Art. 68 Abs. 3 der Verfassung, die Bevorzugung bestimmter Subjekte vorgesehen sein.

4. Der Zugang zu den Leistungen der Gesundheitsfürsorge soll als die Möglichkeit der Nutzung der in dem System garantierten Gesundheitsfürsorge(-leistungen) verstanden werden, was sowohl den Zugang

48 Vgl. Lach, Refundacja leków a równy dostęp do świadczeń opieki zdrowotnej (Arzneimittelkostenrückerstattung und der gleiche Zugang zu der Leistungen der Gesundheitsfürsorge), „Ruch Prawniczy, Ekonomiczny i Socjologiczny” 2014, Heft 1, S. 119-131. 
zum System, als auch den Zugang zu der Gesundheitsfürsorge (bzw. deren Leistungen) für die Systemteilnehmer umfasst.

5. Gegenstand des Zugangs (und der Finanzierung) ist die Gesundheitsfürsorge, deren Bereich in jedem Fall individuell angepasst wird. Die erforderlichen medizinischen Dienst-, Sach- und Begleitleistungen werden in Abhängigkeit von den Fachbewertungen des medizinischen Personals bestimmt.

6. Gleichheit des Zugangs heißt, dass in Bezug auf die Leistungen der Gesundheitsfürsorge weder Quantität (Art und Umfang) noch Qualität (Standard) oder die Voraussetzungen der Gewährung von der materiellen Lage oder dem Grad der finanziellen Partizipation des Leistungsberechtigten (die unterschiedlich sein können) abhängig gemacht werden dürfen.

7. Es ist also verboten, die Rechtsregulation so zu gestalten, dass beim Zugang in ungerechtfertigter Weise aufgrund der materiellen Lage der Bürger differenziert wird. Dies bezieht sich insbesondere auf die Einführung ungerechtfertigter Finanzschranken.

8. Eine Betrachtung des Sozialrechts ergibt, dass die Gesellschaftspolitik einen Chancenausgleich und die Beseitigung übermäßiger Unterschiede in der Lebenssituation verschiedener Personengruppen bezweckt. Daraus folgt, dass einer diesem Ziel dienenden Rechtsregelung von Anfang an die Annahme einer Ungleichheit in Bezug auf die anerkannten Rechte oder die auferlegten Lasten zugrunde liegen muss. Erst im Lichte dieser Annahme kann man die bestehende Regelung bezüglich der angewandten Verteilungskriterien dahingehend bewerten, ob sie objektiv, relevant, verhältnismäßig und gerecht ist. Anschließend kann festgestellt werden, ob die Verteilung von Rechten und Pflichten zwischen den so bestimmten Personengruppen gleich und damit in dem Sinne ist, dass die bestehenden Unterschiede berücksichtigt werden.

9. Unter Berücksichtigung der besonderen Axiologie des Sozialrechtes kann man sagen, dass die Zulässigkeit der Differenzierung sowie der angewendeten Differenzierungskriterien nicht nur mit dem Gerechtigkeits- oder Gemeinwohl-, sondern auch mit dem Solidaritätsgrundsatz begründet werden kann.

10. Bei dem Aufbau von Systemen der sozialen Sicherung sollte der Gesetzgeber nicht nur gewisse axiologische Annahmen berücksichtigen und verfassungsrechtlich bestimmte Ziele verfolgen, sondern auch die rechtlichen und tatsächlichen Möglichkeiten beachten. Deshalb steht 
ihm bei der Wahl bestimmter Lösungen bzw. bei der Schaffung neuer Institutionen ein erheblicher Spielraum zu.

11. Bei einer rechtlichen Beurteilung im Lichte der Programmnormen der Verfassung sind nur solche Regelungen zu beanstanden, die klar und eindeutig im Widerspruch $\mathrm{zu}$ den in diese Programmnormen zum Ausdruck gebrachten Zielen stehen. Wenn die bestehenden Lösungen bedenklich sind, aber das Handeln des Gesetzgebers nicht als willkürlich bezeichnet werden kann, dürfen nur de lege ferenda-Postulate formuliert werden. Die Entscheidungen sind hingegen dem Gesetzgeber mit der durch Wahlen zum Ausdruck gebrachten Legitimation des Souveräns zu überlassen.

12. Die derzeitigen rechtlichen Regelungen bezüglich der finanziellen Belastungen der einzelnen Subjekte (bzw. deren Gruppen) können als umstritten bezeichnet werden. Es ist daher eine Änderung der bestehenden Regelung zu fordern. Dennoch liegt in ihr keine „unbestreitbare und offensichtliche" Verfassungsverletzung. 
